[Highlight]

\title{
纳米尺寸金属氧化物团簇的化学反应性
}

杨金龙

(中国科学技术大学化学与材料科学学院, 合肥 230026)

\section{Reactivity of Nano-Sized Metal Oxide Clusters}

\author{
YANG Jin-Long
}

(School of Chemistry and Materials Science, University of Science and Technology of China, Hefei 230026, P. R. China)

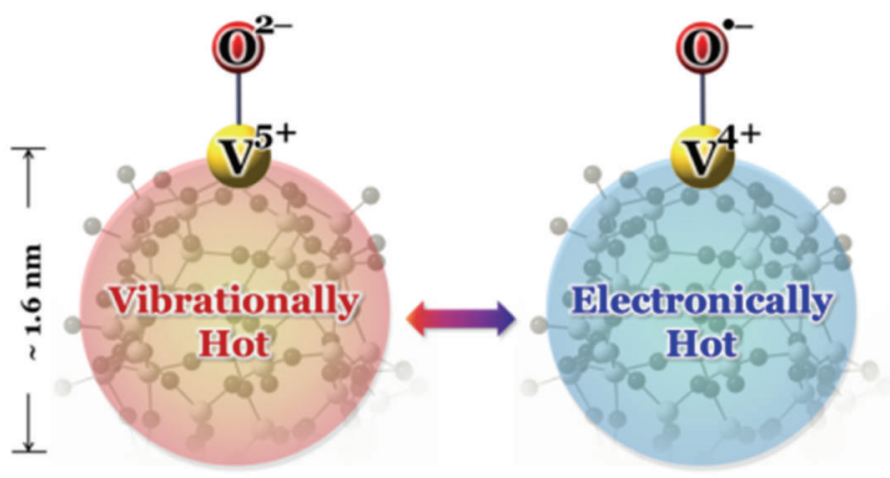

金属氧化物是一类重要的催化剂或催化剂载 体。理性地设计和改进催化剂, 基于对反应活性 位点与反应性构效关系的充分认识。相较于复杂 的凝聚相体系, 气态条件下的金属氧化物团簇具 有元素组分和原子数目确定的特点, 是一类从原 子分子层次上认识催化反应性的重要模型 ${ }^{1-4}$ 。对中 小尺寸金属氧化物团簇 $\left(\mathrm{M}_{x} \mathrm{O}_{y}{ }^{q}\right)$ 的研究发现, “氧缺 陷指数” $(\Delta$, 方程 $(1), m$ 为金属 $\mathrm{M}$ 的最高价态, $\mathrm{M}$ 通常为前过渡金属)为 1 的团簇一般含有原子氧 自由基 $\left(\mathrm{O}^{\bullet-}\right)^{5,6}$ :

$$
\Delta=2 y-m x+q
$$

该自由基在与碳氢化合物、一氧化碳等小分子的 反应中通常表现出优越的化学活性:

$$
\begin{aligned}
& \mathrm{O}^{\bullet-}+\mathrm{RH} \rightarrow \mathrm{R}^{\bullet}+\mathrm{OH}^{-} \\
& \mathrm{O}^{\bullet-}+\mathrm{CO} \rightarrow \mathrm{CO}_{2}+\mathrm{e}^{-}
\end{aligned}
$$

而增加或减少若干个氧原子的团簇 $(\Delta \neq 1)$ 通常呈 现化学惰性。探索氧化物团簇的反应性在何种尺 度呈现体相行为, 即体系中增加或减少若干氧原 子, 体系的性质不发生显著的变化, 对进一步认 识金属氧化物的催化反应性具有重要意义。
最近, 中国科学院化学研究所何圣贵研究员 课题组采用自行设计的高分辨率质谱仪, 成功观 测到氧化物的化学反应性从团簇行为向体相行为 的转变。氧化钒在催化氧化正丁烷制马来酸䣶等 重要工业过程中应用广泛 ${ }^{7.8}$, 研究氧化钒团簇阴离 子 $\left(\mathrm{V}_{2} \mathrm{O}_{5}\right)_{n} \mathrm{~V}_{x} \mathrm{O}_{y}^{-}(n=1-31 ; \Delta=0, \pm 1, \pm 2)$ 与正丁烷的 反应发现, 当 $n<25$ 时, $\Delta=1$ 的 $\left(\mathrm{V}_{2} \mathrm{O}_{5}\right)_{n} \mathrm{O}^{-}$团簇表 现出明显强于其他团簇 $(\Delta \neq 1)$ 的氢抽取反应活 性; 而当 $n>25$ 时, $\Delta=0, \pm 1, \pm 2$ 的团簇的氢抽取 反应活性趋于相当, 由此得出氧化钒纳米粒子的 氢抽取反应活性由团簇行为向体相行为的转变发 生在尺寸为 $1.6 \mathrm{~nm}\left(\sim \mathrm{V}_{50} \mathrm{O}_{125}\right)$ 处。该研究提出, 当 氧化钒纳米粒子的尺寸大于 $1.6 \mathrm{~nm}$ 时, 每个原子 对振动能提供的小部分贡献 $\left(3 k_{\mathrm{B}} T\right)$, 使得整个团簇 的振动能足够高, 同时在强电一声耦合作用驱动 下, 具有低带隙的氧化钒纳米粒子表面发生电子 转移, 生成高活性的原子氧自由基 $\left(\mathrm{O}^{-}{ }^{-}\right)$:

$$
\mathrm{V}^{5+}=\mathrm{O}^{2-}+E_{\mathrm{vib}} \rightarrow \mathrm{V}^{4+}-\mathrm{O}^{\bullet-}
$$

在该活性中心生成机制作用下, 气态条件下的氧 化物纳米粒子的反应活性可以有数量级程度的提 
高。相反, 在带隙较大的氧化钪体系中, 实验发 现尺寸大到 $\mathrm{Sc}_{52} \mathrm{O}_{y}^{-}$的体系仍然表现出团簇行为。

该工作首次从单一质量分辨水平明确提供了 氧化钒体系从团簇行为到体相行为转变的尺度范 围, 证实了氧化物表面热致电子转移生成活性原 子氧自由基的作用机制, 揭示了工业过程中烷烃 活化的机理。该研究成果发表在最近的 Journal of the American Chemical Society 杂志上？

\section{References}

(1) O'Hair, R. A. J.; Khairallah, G. N. J. Cluster Sci. 2004, 15, 331. doi: 10.1023/B:JOCL.0000041199.40945.e3

(2) Böhme, D. K.; Schwarz, H. Angew. Chem. Int. Ed. 2005, 44, 2336. doi: 10.1002/anie. 200461698
(3) Xue, W.; Wang, Z. C.; He, S. G.; Xie, Y.; Bernstein, E. R. J. Am. Chem. Soc. 2008, 130, 15879. doi: 10.1021/ja8023093

(4) Castleman, A. W. Catal. Lett. 2011, 141, 1243. doi: 10.1007/ s10562-011-0670-7

(5) Ding, X. L.; Wu, X. N.; Zhao, Y. X.; He, S. G. Acc. Chem. Res. 2012, 45, 382. doi: 10.1021/ar2001364

(6) Ma, J. B.; Xu, B.; Meng, J. H.; Wu, X. N.; Ding, X. L.; Li, X. N.; He, S. G. J. Am. Chem. Soc. 2013, 135, 2991. doi: 10.1021/ ja311695t

(7) Centi, G.; Trifirò, F.; Ebner, J. R.; Franchetti, V. M. Chem. Rev. 1988, 88, 55. doi: 10.1021/cr00083a003

(8) Weckhuysen, B. M.; Keller, D. E. Catal. Today 2003, 78, 25. doi: 10.1016/s0920-5861(02)00323-1

(9) Zhang, M. Q.; Zhao, Y. X.; Liu, Q. Y.; Li, X. N.; He, S. G. J. Am. Chem. Soc. 2017, 139, 342. doi: 10.1021/jacs.6b10839 